\title{
TIC en el curriculo de matemáticas. Una orientación desde el marco de las politicas y proyectos educativos ${ }^{1}$
}

\author{
Mayra Alejandra Arévalo Duarte ${ }^{2}$ \\ Audin Aloiso Gamboa Suárez ${ }^{3}$
}

Fecha de recepción: 12 de marzo de 2015

Fecha de revisión: 20 de mayo de 2015

Fecha de aprobación: 5 de junio de 2015

\section{Resumen}

El presente artículo muestra una reflexión sobre los criterios que orientarán la integración de las TIC en el currículo de matemáticas desde el marco de las políticas y proyectos educativos TIC desarrollados en el territorio colombiano. El análisis se desarrolló a partir de la identificación de fuentes primarias y secundarias de información, la fundamentación del sistema de categorías y el diseño del instrumento para la organización y sistematización de la información. Los resultados producto del proceso de análisis de estas fuentes configuran algunos aspectos para orientar

1 Artículo de reflexión derivado de una investigación titulada "Competencias TIC de los docentes de Matemática en el enfoque del conocimiento tecnológico-pedagógico del contenido", avalado por el grupo de investigación en estudios sociales y pedagogía para la paz (GIESPPAZ, categoría B en Colciencias) de la Universidad Francisco de Paula Santander, Cúcuta.

2 Docente investigadora de la Universidad Francisco de Paula Santander, Cúcuta (Colombia). Magister en Educación de la Pontificia Universidad Javeriana de Bogotá. Doctora en Educación y TIC de la Universidad de Salamanca, España. Dirección de correspondencia: avenida Gran Colombia No. 12E-96, barrio Colsag, Cúcuta, Norte de Santander, departamento de Pedagogía, segundo piso edificio CREAD. Teléfono de contacto: 5776655 ext. 143. Correo electrónico: mayiarevalo@gmail.com

3 Docente investigador de la Universidad Francisco de Paula Santander, Cúcuta. Magister en Pedagogía (UIS), doctor en Ciencias de la Educación (RudeColombia, CADE Universidad de Cartagena). Dirección de correspondencia: avenida Gran Colombia No. 12E-96, barrio Colsag, Cúcuta, Norte de Santander, departamento de Pedagogía, segundo piso edificio CREAD. Teléfono de contacto: 5776655 ext.143. Correo electrónico: docaudin@gmail.com 
la integración de las TIC en el currículo de matemáticas. Finalmente, se concluye que las políticas y proyectos desarrollados en torno a las TIC son un insumo fundamental para construir las bases teóricas y metodológicas de la incorporación de las TIC en el sistema educativo, y para caracterizar el contexto que le permitirá a las instituciones educativas comprender las exigencias del Estado y la sociedad para plantear currículos pertinentes y viables según las metas y propósitos de la educación en Colombia.

\section{Palabras clave:}

Políticas TIC, proyectos educativos TIC, currículo, educación matemática.

\section{TIC in the mathematics curriculum a guidance from the framework of political and educational projects}

\section{Abstract}

The study had as an objective to define criteria to guide the integration of TIC in the mathematics curriculum from the context of the policies and educational projects TIC developed in Colombia. It grew out of the research methodology literature which identified primary and secondary sources of information that formed the theoretical framework, the foundation of the category system and the design of the instrument for the organization and systematization of information. The results, a product of the analysis process of these sources, meet each of the objectives and outline some ways to guide the integration of TIC into the mathematics curriculum. Finally, we conclude that policies and projects developed around TIC is a fundamental input to build the theoretical base and methodologies of the incorporation of TIC into the education system and to characterize the context that will allow educational institutions to understand the requirements of the state and society to propose relevant and viable curriculum according to the goals and purposes of education in Colombia. 


\section{Keywords:}

TIC policies, TIC educational projects, curriculum, mathematics education.

\section{Tic na matemática currículo. A orientação do quadro de políticas e projectos educativos}

\section{Resumo}

Este artigo apresenta uma reflexão sobre os critérios para orientar a integração das TIC no currículo de matemática a partir do contexto de políticas de TIC e projetos educacionais na Colômbia. A análise foi desenvolvida a partir da identifición de fontes primárias e secundárias de informação, a lógica do sistema de categorias e ferramenta de projeto para a organização e sistematização de informações. Os resultados do produto da análise destas fontes compõem alguns aspectos para orientar a integração das TIC no currículo de matemática. Por fim, conclui-se que as políticas e projetos desenvolvidos em torno das TIC é um insumo fundamental para construir as bases teóricas e metodológicas para a incorporação das TIC no sistema de ensino e caracterizar o contexto que irá permitir que as instituições de ensino para compreender a exigências do Estado e da sociedade para pedir currículo relevante e viável de acordo com as metas e objetivos da educação na Colômbia.

\section{Palavras-chave:}

Políticas TIC, programas educacionais de TIC, currículo, educação matemática.

\section{Introducción}

La incorporación de las TIC en la sociedad, ha llevado a que los países establezcan un sistema de monitoreo y control a partir de indicadores que evalúan el avance de los mismos en este medio. Este sistema oficialmente presentado como políticas, permite que los países analicen sus logros, fracasos y se propongan metas que puedan ser comparadas a nivel nacional e internacional, con el fin de determinar el 
grado de compromiso y preparación para afrontar la inclusión digital. Las políticas construidas en materia de TIC han determinado que la educación es un elemento fundamental para trascender hacia la sociedad de la información, y ponen de manifiesto que las TIC son un factor importante para lograr esta trascendencia y formar ciudadanos que respondan a las necesidades de la sociedad contemporánea. En este sentido, se reconoce que el uso de las TIC puede mejorar la calidad de la educación, innovar en las prácticas educativas, enriquecer los aprendizajes, desarrollar competencias personales, profesionales y también se espera que su inclusión en la escuela reduzca la brecha digital, la pobreza, y contribuya con el desarrollo social y económico de los países.

Estos retos demandan de los sistemas educativos nuevas respuestas en modelos que integren además de estas características socio-culturales las tecnologías, y prepare a los ciudadanos para enfrentar las exigencias que requiere la inclusión digital. Exigencia que debe ser atendida no solo desde una necesidad de tipo cuantitativo (más computadores, Internet, herramientas tecnológicas) sino como un desafío de tipo cualitativo, donde se debe repensar el sentido, la organización de la escuela y del currículo escolar, dado que muchos problemas de incorporación de las TIC obedecen a estos hechos que se describen como: falta de actualización de los sistemas y del currículo, y se expresan como problemas de burocratización de la administración, de rutinización de las prácticas escolares, de obsolescencias de los contenidos curriculares, e ineficiencia de los resultados finales (Aguerrondo, 2007). En este sentido, los sistemas educativos deben propender por desarrollar iniciativas didácticas, innovadoras, flexibles, independientes de currículos rígidos, convencionales, desarticulados de las competencias digitales, que tengan como eje central responder a los desafíos de tipo cualitativo, proporcionando paralelamente los recursos tecnológicos necesarios para atender los retos cuantitativos de forma pertinente.

En torno a estas situaciones se plantea la presente reflexión, la cual pretende acercarse a la integración curricular de las TIC en matemáticas desde un contexto político, guiado por el siguiente interrogante: icontribuyen las políticas y proyectos TIC desarrollados en Colombia a integrar las tecnologías al currículo de matemáticas?

Este trabajo se inclina por un enfoque socio político educativo, porque los lineamientos, los recursos, los aspectos y necesidades socio culturales que deben estar presentes en la planeación educativa, se encuentran caracterizados en las políticas emanadas por el Estado, además porque el Estado es el primer responsable en promover y facilitar desde sus políticas los medios para que la integración de 
las tecnologías sea posible en los diferentes sectores sociales, especialmente en el contexto educativo. Una vez determinados los parámetros y compromisos por parte del Estado, los siguientes responsables de la integración de las TIC son los docentes, al planear, organizar y diseñar currículos y prácticas educativas innovadoras mediadas por el uso de estos recursos.

Al respecto, las afirmaciones de este texto pretenden promover la reflexión de estos roles docentes, proporcionando elementos que faciliten la integración de las TIC en el currículo de matemáticas, desde el marco de las políticas y proyectos educativos TIC desarrollados en el territorio colombiano, a partir de la consecución de propósitos específicos como el de categorizar las políticas y proyectos que se han implementado en el territorio colombiano para la integración de las TIC, identificar elementos que permitan su integración contextualizada en el currículo de matemáticas y finalmente establecer si existe relación entre los elementos que determina la norma educativa y los elementos encontrados en las políticas y proyectos educativos TIC para el diseño curricular en matemáticas.

\section{El curriculo y las políticas educativas}

La incorporación de las tecnologías en el Estado colombiano se inscribe dentro de un marco social de referencia denominado sociedad de la información y del conocimiento (SIC), término acuñado por los países desarrollados para designar el auge que ha tenido la integración de las TIC en todos los campos de la actividad humana. La necesidad a nivel mundial de establecer lineamientos para el acceso, uso y apropiación pertinente de estos recursos tecnológicos, ha llevado a que diferentes países del mundo se reúnan en torno a este tema y se definan parámetros realizables en las diferentes zonas geográficas del mundo, con el fin de crear una sociedad centrada en la persona, con igualdad de acceso a la información, que promueva el desarrollo sostenible y la calidad de vida equitativa de las comunidades.

Con este propósito fue celebrada la Cumbre Mundial de la Sociedad de la Información, donde se definió este modelo de sociedad y se establecieron los fundamentos para que los países del mundo definieran sus políticas en torno a este tema. En este marco internacional, Colombia establece sus lineamientos para la incorporación de las TIC y visiona el modelo de sociedad de información que necesitan los colombianos, con la participación activa de la población, los sectores públicos, privados y la dirección del gobierno nacional. El modelo de sociedad que propone Colombia se define como un 
modelo que se apoya en el uso y apropiación adecuada de las TIC para lograr el crecimiento productivo y el progreso económico y social del país (Ministerio de Tecnologías de la Información y las Comunicaciones, 2008). Esta visión de modelo le permitió al país fundamentar y consolidar la política nacional sobre tecnología, denominada Plan Nacional de Tecnologías de la Información y las Comunicaciones, en adelante PNTIC.

EI PNTIC plantea una serie de propósitos fundamentales que se proyectan desarrollar a corto y largo plazo. Los propósitos a corto plazo se establecen como respuesta a la integración masiva y acelerada de las TIC en la sociedad, y se especifican en mejorar el acceso a la infraestructura, ayudar a la incorporación de las TIC y consolidar el proceso de gobierno en línea. Los propósitos fundamentales a largo plazo se especifican en mejorar la inclusión social, aumentar la competitividad haciendo uso eficiente y productivo de las TIC y posicionar a Colombia en los primeros lugares a nivel latinoamericano en materia de TIC. También se define una matriz de ocho ejes, divididos en dos grupos, que contribuye a lograr estos propósitos. En el primer grupo se encuentran los ejes transversales (Comunidad, Gobierno en línea, Investigación-desarrollo-innovación, Marco normativo-regulatorio e incentivos), que tienen como función plantear acciones que permean los sectores considerados importantes. El segundo grupo lo conforman los ejes verticales (Educación, Salud, Justicia y Competitividad empresarial), encargados de contextualizar estas acciones. A partir de esta política se han consolidado nuevos programas, como el Plan Vive Digital Colombia, y se han renovado los existentes, los cuales se venían desarrollando a través del tiempo, pero que no se encontraban sistematizados en una política que los reuniera y orientara su curso.

El Plan Vive Digital Colombia fue lanzado por el Ministerio TIC en el año 2010 para impulsar la masificación del uso de Internet, multiplicar el número de conexiones a la red, conectar las MIPYMES y los hogares colombianos (MINTIC, 2011). Para el desarrollo de estos propósitos el plan asume el modelo de ecosistema Digital (Kim, Kelly y Raja, 2010), el cual busca estudiar y analizar todos los componentes que intervienen en la masificación del uso de Internet para diseñar estrategias que permitan incentivarlo. Estos componentes se agrupan en Oferta (Infraestructura y Servicios) y Demanda (Aplicaciones y Usuarios), e interaccionan dependiendo uno del otro, es decir, a mayor uso del Internet por parte de la población, mayor demanda de infraestructura y servicios, lo que genera una apertura de los mercados, crecimiento de la oferta y mejora de los precios. Al disminuir los precios, más usuarios se integrarán a este ecosistema, generando una demanda de aplicaciones y contenidos, lo que denomina el Ministerio TIC como un círculo 
virtuoso que conlleva al modelo de sociedad de la información que se propone desarrollar el país.

En lo que respecta al sector educativo, el Estado colombiano, junto con el Ministerio de Educación Nacional y el Ministerio de Tecnologías de la Información y las Comunicaciones, también han venido desarrollando diferentes programas y proyectos en materia de TIC, los cuales han sido el fundamento de las políticas actuales. El Programa Estratégico de uso de Medios y Tecnologías de la Información y la Comunicación (MTIC) lanzado en el 2002, ha integrado las expectativas de estos ministerios, proponiendo así el desarrollo de competencias en los maestros para que incorporen las TIC de forma pedagógica y didáctica en los procesos de enseñanza a nivel de la educación preescolar, básica, media y superior. Este programa establece líneas de acción para apoyar las políticas del MEN en cuanto a calidad educativa, cobertura y eficiencia a través de la dotación de computadores, conectividad, desarrollo de contenidos digitales y la definición de procesos y estándares para el uso y apropiación de las tecnologías en la educación. Para el cumplimiento de estos propósitos, el programa ha desarrollado una serie de proyectos que han permitido la apropiación de las TIC en dos campos: el campo personal (proyecto: Compartel, Computadores para educar, y A que te cojo ratón) y el campo profesional (proyecto: Temáticas y PlanEsTIC).

En el campo específico de las matemáticas, la incorporación de las TIC al currículo se originó a partir de cuatro situaciones que lo provocaron, la primera corresponde a los resultados arrojados por diferentes estudios donde se muestra el bajo rendimiento de los estudiantes en esta área, atribuido a la práctica de un currículo de matemáticas, que pone en evidencia enseñanzas tradicionales, donde el estudiante solo hace alarde de su memoria para lograr el aprendizaje y no de procesos mentales superiores que implica el aprendizaje de las matemáticas.

La segunda situación fue la necesidad de construir los lineamientos curriculares para el área de matemáticas que se publicaron en el año 1998, y abordan la problemática descrita anteriormente a partir de la descripción de tres aspectos básicos como son: los procesos generales, los conocimientos básicos y el contexto (Ministerio de Educación Nacional, 1998).

La tercera situación, corresponde a la reflexión que se hizo del documento Informe Mundial sobre la Educación: los docentes y la enseñanza en el mundo en mutación, publicado por la Unesco en 1998. En este informe, se plantea que los grandes retos educativos están configurados desde una formación para todos y para toda la vida, donde las tecnologías juegan un papel importante junto con 
la formación docente como eje central para que se produzcan las transformaciones necesarias. También se reflexiona sobre la incidencia de las tecnologías en el campo educativo y las tendencias de los procesos de enseñanza, abordando situaciones como: la posibilidad de que dicho fenómeno pueda incidir en la mejora de la calidad de la enseñanza, los cambios que supone en el papel y funciones del educador; las consecuencias tanto a nivel económico como social que trae consigo; la posibilidad de ser un factor generador de diferencias significativas por parte de los educandos, entre otros (Unesco, 1998).

La cuarta situación corresponde al proyecto Nuevas Tecnologías y Currículo de Matemáticas que se desarrolló este mismo año (1998) en el territorio colombiano, y contó con el apoyo de la OEA, de expertos colombianos, de Gran Bretaña, México, Chile, de facultades e instituciones de educación básica, media y superior; su objetivo era conocer las experiencias significativas que se habían desarrollado en torno a la incorporación de las tecnologías en el sector educativo y determinar criterios para re-orientar su incorporación, trabajando específicamente con las calculadoras y el software CABRI.

A partir de estos antecedentes, se piensa a nivel nacional en incorporar las tecnologías al currículo de matemáticas, consolidándose en el año 2000 el proyecto Incorporación de Nuevas Tecnologías al currículo de Matemáticas de la Educación Básica Secundaria y Media, liderado por el Ministerio de Educación Nacional, en coordinación con diferentes instituciones del país y un grupo de expertos, donde se obtuvieron resultados, entre otros, que determinaron los referentes pedagógicos y didácticos para orientar la incorporación de las TIC en el currículo de matemáticas (MEN, 2001).

Estos referentes reconocen como contexto los cambios que han acontecido en la sociedad del siglo XXI, dado los adelantos tecnológicos y científicos que han resignificado la función de la escuela al pasar de ser el principal canal de formación a reconocer que existen otros canales en los que se está formando la sociedad; la función del docente al pasar de ser el que dirige y controla los procesos de aprendizaje, al que genera ambientes para que el estudiante construya su aprendizaje; la función del estudiante al pasar de ser receptor de la información a ser activamente constructivo en su formación; la función de los procesos de enseñanza y aprendizaje, al pasar de estar centrados en el rol del profesor y de los contenidos, a centrarse en las formas de aprendizaje de los estudiantes y en el desarrollo de competencias para recoger, procesar e interpretar la información que reciben del medio, y finalmente, el conocimiento deja de ser estático y escaso a ser una burbuja que crece aceleradamente y se difunde fácilmente por los medios. 
En el contexto de estos cambios, se propone la incorporación de las TIC en el currículo de matemáticas, ya que son las mismas tecnologías que lo han propiciado. Un currículo que contextualice el conocimiento sobre el qué, el cómo y el cuándo de estos cambios, permitirá abordar esta realidad desde un contexto escolar, comprender el comportamiento humano, procesar la información que se recibe con la que se tiene, usar nuevas herramientas tecnológicas y promover la construcción del conocimiento en una sociedad que cambia y busca el progreso científico y tecnológico. Para tal fin, el enfoque desde el cual se comprenden estos fenómenos que evolucionan a partir de la integración de las tecnologías se realiza desde la construcción del conocimiento a partir de las teorías de Vygostky (1979), su relación con la mediación de los instrumentos tecnológicos (Verillon y Rabardel, 1995), la didáctica de las matemáticas apoyados en la teoría de Brousseau (2007) y los fundamentos sobre el currículo en matemáticas (Rico, 1998).

\section{Criterios curriculares para la integración de las TIC en educación matemáticas}

Asumiendo la autonomía que delega el Ministerio de Educación Nacional a las instituciones educativas para diseñar el currículo de las áreas (MEN, 1994), y teniendo como base la normativa establecida para guiar el diseño curricular, el MEN espera que sean los docentes los que elaboren el currículo, ya que son los que tienen los conocimientos y capacidades para contextualizar esta normativa y las políticas educativas a la realidad de sus instituciones educativas.

Desde esta perspectiva, se plantea una integración curricular de las TIC a partir de un marco educativo con un enfoque constructivista que integra elementos socio históricos y culturales dados por las políticas TIC, y elementos pedagógicos y didácticos encontrados en los proyectos educativos TIC desarrollados en el territorio colombiano. Los elementos socio-históricos y culturales permiten reconocer que existe un contexto que está en constante evolución, donde se forman e interactúan los sujetos y se determinan las funciones educativas de la escuela. Los elementos pedagógicos y didácticos definen los métodos de apropiación de las TIC y la acción pedagógica del profesor para desarrollar un proceso de mediación donde se produzca el aprendizaje. Asimismo, la construcción del conocimiento se produce a través de la interacción del sujeto con los objetos que tienen una connotación histórica y social, los cuales son explorados a partir de las actividades culturalmente 
organizadas por los profesores, donde las herramientas TIC juegan un papel importante puesto que son creadas por la misma sociedad e introducidas a partir de un proceso de interacción (Vygotsky, 1979). En este sentido, se considera que integrar las TIC al currículo de matemáticas constituye un proceso de articulación de los fines educativos, la apropiación de las herramientas TIC y la acción pedagógica del profesor para conformar un todo que es el currículo, que tiene como fin último, el aprendizaje y el desenvolvimiento de los estudiantes en contextos multidisciplinares. Por lo tanto, cuando existe realmente una integración curricular de las TIC, estas deben tornarse invisibles en la práctica, para hacer visible el aprendizaje y el desarrollo de competencias de los estudiantes.

En este sentido, se establece que la norma educativa concibe al currículo como un instrumento de desarrollo social e institucional con una carga cultural y un compromiso de la comunidad en general, al definirse como un conjunto de criterios, planes de estudio, metodologías y procesos que contribuyen a la formación integral y a la construcción de la identidad cultural nacional, regional y local, incluyendo también los recursos humanos, académicos y físicos para poner en práctica las políticas y llevar a cabo el proyecto educativo institucional (Ministerio de Educación Nacional, 2002).

Dado este enfoque, en cuanto al análisis de las políticas y proyectos educativos TIC se tiene que, en la actualidad existen dos tipos de políticas a nivel nacional que engloban todo el plan de integración de las TIC y que determinan los lineamientos que se deben seguir para hacer efectiva esta integración, dichas políticas se categorizaron como políticas públicas en materia de TIC y política educativa en materia de TIC. Fueron fijadas por el gobierno siguiendo el modelo de sociedad que se estableció en la Cumbre Mundial de la Sociedad de la Información que se encuentra actualmente en vigencia. En la descripción de estas políticas se identificaron elementos relevantes que las describen y que se toman como componentes para su análisis, estos son: contenidos, rol del Estado, retos e implementación.

Respecto a los proyectos educativos TIC, se determinaron dos subcategorías dados los niveles de integración que se encontraron: una integración personal o alfabetización básica y una integración profesional que corresponde al uso de las tecnologías en la práctica docente. En estos proyectos emergen los siguientes componentes que los describen: gestión tecnológica, formación docente y resultados. También se pone en evidencia que estos proyectos de incorporación de las TIC contemplan dentro de sus principios la formación docente basada en el desarrollo de competencias, la cual es orientada por grupos de expertos asignados por el MEN, con contenidos 
específicos que promueven el uso de las TIC y con metodologías incluyentes y flexibles. Sin embargo, es importante resaltar que los contenidos abordados para la formación son generales, no existe una especificidad dada la naturaleza de cada asignatura del currículo para que los maestros según su saber específico profundicen en sus formas de enseñanza mediadas por recursos tecnológicos. En el área de matemáticas, a nivel nacional se ha desarrollado un solo proyecto denominado Proyecto de Incorporación de Nuevas Tecnologías al currículo de Matemáticas de la Educación Básica Secundaria y Media, que contempla esta situación y que pone en evidencia la necesidad de un conocimiento profesional docente consolidado en la teoría y en la práctica para diseñar y desarrollar currículos con saberes específicos que contemplen procesos de enseñanza y aprendizaje mediados por las TIC, y procesos de evaluación para conocer avances y vislumbrar transformaciones educativas.

Una vez explicitada esta relación, se procede a definir los criterios que orientan la integración de las TIC al currículo de matemáticas en el marco de las políticas y proyectos educativos TIC desarrollados en el territorio colombiano. La siguiente Tabla 1, se toma como base para definir estos criterios, para cada elemento descrito se determina un elemento que tiene la función de conjugarlos en el contexto de las TIC, con el fin de planificar y usar sus potencialidades dentro de un currículo.

Tabla 1. Criterios curriculares para la integración de las TIC

\begin{tabular}{l|l|l}
\hline $\begin{array}{l}\text { Elementos curriculares } \\
\text { según el MEN }\end{array}$ & $\begin{array}{l}\text { Elementos curriculares } \\
\text { implícitos en las políticas y y } \\
\text { proyectos TIC }\end{array}$ & $\begin{array}{l}\text { Elementos fundamentales para } \\
\text { la integración de las TIC al } \\
\text { currículo de matemáticas }\end{array}$ \\
\hline \multirow{2}{*}{$\begin{array}{l}\text { Planes de estudio } \\
\text { Contenidos } \\
\text { Metodología } \\
\text { Evaluación }\end{array}$} & \multirow{2}{*}{ Desarrollo del conocimiento } \\
integral & $\begin{array}{l}\text { Fines de desarrollo perso- } \\
\text { nal y de aprendizaje }\end{array}$ & \\
\hline \multirow{2}{*}{ Recursos } & $\begin{array}{l}\text { Rol del estudiante } \\
\text { Rol del profesor } \\
\text { Rol de la escuela }\end{array}$ & \multirow{2}{*}{\begin{tabular}{l} 
Realidad institucional \\
\hline
\end{tabular}} \\
\hline
\end{tabular}




\begin{tabular}{l|l|l}
\hline $\begin{array}{l}\text { Construcción de } \\
\text { identidad }\end{array}$ & $\begin{array}{l}\text { Fines culturales } \\
\text { Fines políticos y morales } \\
\text { Fines sociales }\end{array}$ & Reconocimiento del contexto \\
\hline \multicolumn{2}{l}{ Planta física y materiales tecnológicos } & Infraestructura \\
\hline
\end{tabular}

Los elementos que se determinan como: desarrollo del conocimiento, realidad institucional, reconocimiento del contexto e infraestructura, se consideran criterios fundamentales desde su función, para la integración de las TIC al currículo de matemáticas. Para cada uno de estos elementos se establece un orden de presentación que guía la planificación y diseño curricular según la función que desempeñan. Por lo tanto, se considera que el proceso de integrar las TIC al currículo de matemáticas, debe iniciar con un reconocimiento del contexto seguido del reconocimiento de la realidad institucional, con estos dos elementos se tiene un panorama global sobre la misión y la visión que debe contemplar el currículo, además de los indicadores a los que debe responder. Pasados estos dos procesos, sigue el reconocimiento de la infraestructura informacional, la cual evidenciará en qué nivel de integración se puede realizar la incorporación de las tecnologías y finalmente, el desarrollo del conocimiento donde se construirá el plan de estudios específico para el área articulando los conocimientos que se pueden desarrollar con el uso de las tecnologías, los tiempos y los procesos evaluativos que permitirán la retroalimentación. Este orden facilita reconocer los elementos que se deben tener presente previo a la construcción del currículo y los elementos que cambian o se transforman según la manifestación de estos primeros.

\section{Descripción de los elementos fundamentales para la integración curricular de las TIC en matemáticas}

Criterio 1 - Reconocimiento del contexto. Se considera importante que a la hora de diseñar un currículo se tome como base el contexto que establecen las políticas, normativas y proyectos que se hayan desarrollado en materia de TIC, ya que estos documentos orientan el alcancen que deben tener los currículos, los mínimos a los que deben responder y los ámbitos en los que se deben comprometer las instituciones educativas y los docentes. Los impactos de estas políticas y proyectos darán un panorama global sobre las experiencias significativas logradas que se pueden replicar, los retrocesos que se deben evitar y las transformaciones que se deben 
realizar al interior de los procesos educativos. Por lo tanto, el reconocimiento del contexto conlleva a una revisión de las normativas propuestas por el MEN para la construcción de currículos, el análisis de las políticas TIC para el "re-conocimiento" de indicadores tecnológicos y educativos con los que se puede comprometer la institución y una socialización de los proyectos educativos desarrollados a nivel nacional para evaluar y apropiar procesos pedagógicos y didácticos exitosos en materia de TIC.

Criterio 2 - Realidad institucional. A la hora de organizar y diseñar un currículo las instituciones educativas deben "re-conocer" su realidad, es decir, evaluar al recurso humano con el que cuentan como docentes, administrativos docentes, estudiantes y otros administrativos, con el fin de asignar pertinentemente las funciones a desarrollar. La evaluación de los docentes permitirá reconocer sus fortalezas y oportunidades de mejora con lo que se evidenciará las necesidades formativas y se proyectarán los planes de formación continua, la evaluación de los estudiantes confirmarán los planes de formación de los maestros, las transformaciones que se deben realizar al interior de los procesos educativos y los énfasis disciplinares que se deben fortalecer, la evaluación del personal administrativo permitirá conocer la efectividad del uso y apropiación de las TIC para la comunicación interna y externa y las posibles transformaciones a realizar. En este proceso también es importante revisar y evaluar los currículos desarrollados anteriormente como precedentes para el diseño de los currículos que integran las TIC.

Criterio 3 - Infraestructura informacional. Para una integración pertinente de las TIC en el currículo, se debe realizar un diagnóstico previo de la planta física y los recursos tecnológicos con los que cuenta la institución, donde se evidencie con datos claros el estado actual de estos recursos, la cantidad y la disponibilidad. También es importante conocer si existen comités para la prestación de servicios técnicos-operativos y académicos, es decir, si la institución educativa cuenta con personal técnico especializado para atender las dudas operativas-técnicas y los interrogantes pedagógicos y didácticos emergentes de las prácticas educativas de maestros y estudiantes. Esta información debe proveerse a los maestros antes de iniciar la organización y construcción del currículo con el objetivo de prever los tiempos y los niveles de integración de las TIC en el área.

Criterio 4 - Desarrollo del conocimiento. En este criterio se propone la construcción del plan de estudios a partir del saber específico en este caso las matemáticas, por lo tanto, los contenidos, objetivos, metodología y evaluación deben construirse a partir de la naturaleza del conocimiento matemático que implica su 
reconocimiento dentro de una cultura que tiene carácter histórico y que es mediada por el uso de instrumentos que proporcionan cercanía a sus avances y a los desarrollos tecnológicos de la sociedad. También se deben contemplar las teorías pedagógicas y sociológicas porque el conocimiento matemático es construido socialmente y está influenciado por el sistema de valores, prácticas sociales, políticas, económicas y su utilidad práctica se da sobre el medio social y natural sobre el cual se ha conjeturado e inferido. Debe integrar teorías de enseñanza y aprendizaje que propendan por un conocimiento matemático relacional que establezca conexiones con otros saberes, que desarrolle la creatividad, la invención, la lógica, la capacidad de razonamiento y organización del pensamiento para interactuar y construir el propio conocimiento relacionándolo con los conocimientos previos donde se contemplan las necesidades formativas, cognitivas, afectivas y sociales.

\section{Conclusiones}

De acuerdo con estos resultados, se considera que debe existir un reconocimiento de las políticas y proyectos educativos para la incorporación de las TIC previo a la construcción del currículo de cualquier área, para el caso específico de las matemáticas, se tiene que, aunque esta construcción y organización curricular está orientada por documentos oficiales emanados del MEN, también debe existir una contextualización que se realiza a partir de la integración de elementos culturales, cognitivos, axiológicos y sociales que se encuentran presentes en las políticas y proyectos TIC y que permiten ser integrados como un todo en el diseño curricular, con el fin de desarrollar prácticas educativas acordes a la realidad social y a las necesidades institucionales.

Las políticas evidencian que la incorporación de las TIC en el sector educativo hace referencia básicamente al uso del computador e Internet. A nivel nacional solo se conoce un proyecto significativo de incorporación de la tecnología como es la calculadora gráfica TI-92 para el aprendizaje de las matemáticas. Este proyecto tuvo gran impacto y aceptación por parte de la comunidad educativa en general, pero duró lo que tardó su desarrollo, y en la actualidad no existen las réplicas de estas prácticas. Por lo tanto, deben existir estrategias de seguimiento y continuidad de estos proyectos, pues se evidencia que el uso de las TIC está determinado por la duración de los proyectos, también se recomienda que las instituciones educativas conformen equipos de trabajo que lideren este proceso, que estén integrados por 
docentes entusiastas y competentes tecnológicamente para que motiven, desarroIlen y regulen actividades académicas en torno a este tema y creen una cultura institucional de uso y apropiación de las TIC.

Frente al rol del Estado ante estas situaciones, se reconoce su compromiso por integrar las tecnologías dada las normatividades, políticas y proyectos existentes en torno a este tema, sin embargo, por los indicadores propuestos y los programas desarrollados hasta el momento, se puede afirmar que esta incorporación se encuentra en un nivel básico dedicado en un alto porcentaje a la dotación de infraestructura y a los procesos de cobertura, sin avanzar significativamente en la incorporación pedagógica y didáctica y en el mejoramiento de los procesos de calidad educativa dados estos recursos tecnológicos.

En cuanto a los elementos que permiten la integración contextualizada de las TIC en el currículo de matemáticas, se tiene que en las políticas y proyectos TIC existen, aunque implícitamente, criterios que contribuyen a plantear un currículo pertinente integrando estas tecnologías, los cuales se pueden categorizar como finalidades culturales, sociales, políticas, de desarrollo, de conocimiento y en algunos casos de contenido y de procesos evaluativos. Los fines sociales y culturales que se plantean en las políticas están asociados a la función de la escuela, y por lo tanto pueden ser incorporados en el diseño curricular para que su desarrollo y evaluación se convierta en un proceso institucional fundamental y de importancia para la comunidad educativa.

Ahora, para que el uso de las TIC sea consolidado como una cultura institucional, debe propiciarse desde varios ámbitos su integración con una formación del recurso humano desde docente y estudiantes hasta el personal administrativo de la institución, esta formación debe ser estratégicamente evidenciada en la planeación, las prácticas educativas y los procesos de aprendizaje de los estudiantes, debe haber una gestión administrativa, que además de visionar la importancia de la integración de las TIC, genere las condiciones técnicas, tecnológicas y operativas necesarias para incorporar, regular y mantener en buen estado estos recursos y genere prácticas comunicativas institucionales internas y externas haciendo uso de los mismos. Deben definirse los roles actuales que deben asumir los docentes y estudiantes para que la incorporación de las tecnologías sea exitosa, estos roles demandan de un crecimiento personal y profesional, tanto en el saber específico como en el saber pedagógico y didáctico, para afrontar los retos, obstáculos, aciertos y desaciertos, para no desmayar en el intento y para que el uso de estos recursos no sean una moda sino que se conviertan en una cultura institucional del buen uso de las TIC. 
Respecto a la relación de los elementos evidenciados en las políticas y proyectos TIC y los que propone el MEN para el diseño curricular, se pudo establecer una comparación directa entre estos aspectos, ya que se corresponden y complementan porque contienen lineamientos que pueden ser categorizados dentro de los elementos que se establecen como fundamentales para la construcción curricular, lo que pone de manifiesto que estas políticas y proyectos han sido articuladas con las políticas educativas y permiten la contextualización del currículo.

Con el análisis de estos documentos y las relaciones que se establecieron entre los elementos que los constituyen, se da respuesta al objetivo general, que consiste en definir criterios que orienten la integración de las TIC al currículo de matemáticas, al respecto se tiene que existen elementos fundamentales que no pueden ser negociados a la hora de "re-pensar" y construir un currículo que integre las TIC, estos se describen como contexto, sistema educativo e infraestructura, ya que son los que fundamentarán posteriormente el desarrollo de los planes de estudios y el logro o no de los objetivos académicos propuestos. Sin embargo, se concluye que estos modelos no son camisa de fuerza, que sus elementos son relacionales y se pueden transformar o cambiar según los contextos e interés institucionales, lo que se considera importante es que la institución educativa integre a toda su comunidad en la incorporación de las TIC al currículo, que en conjunto planeen estrategias para hacer efectiva esta integración y que estén especificadas con tiempos en el currículo para que puedan ser aplicadas, controladas y evaluadas.

Si no existe la infraestructura para hacer eficiente la integración de las TIC, se debe iniciar con mecanismos básicos de gestión de recursos tecnológicos ante el MEN o los respectivos organismos, ya que en las políticas se evidenció que existen programas que están favoreciendo la dotación de equipos e Internet para las instituciones educativas. Si la necesidad es de formación del personal docente, también se debe hacer la respectiva gestión, ya que existen programas de capacitación ofrecidos por el MEN para formar a los docentes en la integración de las TIC a su labor personal y profesional, y también existen instituciones de educación superior especializadas en este tema que han sido encargadas para brindar acompañamiento y orientar a las instituciones que lo necesiten. Igualmente, para el caso específico de las matemáticas, la integración de las TIC demanda de los educadores un conocimiento sólido sobre estructuras conceptuales del área, del conocimiento didáctico y de prácticas adecuadas para organizar el conocimiento matemático para darlo a conocer a través de las tecnologías, considerando el uso crítico y no desmedido de 
estos recursos tecnológicos y determinando espacios y tiempos para que se integren de forma pedagógica, planificada y no al azar.

En cuanto a la pregunta: ¿contribuyen las políticas y proyectos TIC a integrar las tecnologías al currículo de matemáticas? Después del recorrido bibliográfico realizado y el análisis llevado a cabo, se puede afirmar que las políticas y proyectos desarrollados en torno a las TIC han sido un insumo fundamental para construir las bases teóricas y metodológicas de la incorporación de las TIC en el sistema educativo. Sin embargo, su contribución depende no de las políticas y proyectos en sí mismos, sino del reconocimiento que se haga de estas y su aplicación para el diseño curricular. Pueden existir currículos que no se fundamenten en estos documentos, pero está claro que no dan respuesta a un contexto y a unas necesidades sociales que se encuentran categorizadas en estas fuentes documentales. La identificación de elementos, procesos y recursos descritos en estas políticas y proyectos TIC, le facilita a las instituciones educativas comprender las exigencias del Estado y la sociedad para plantear currículos pertinentes y viables según los recursos (físicos, humanos, tecnológicos) con los que cuente la institución, con los compromisos del Estado y con las metas y propósitos de la educación en Colombia.

Desde una perspectiva general, las políticas proporcionan la visión, son la guía, la orientación, y los proyectos proporcionan el ejemplo, la práctica, se convierten en el método, el cual es imprescindible a la hora de diseñar un currículo, ya que no se construye desde cero, sino desde una base confiable que ha sido validada a nivel nacional. Desde una perspectiva específica, las políticas TIC describen en su contenido elementos sociales, culturales y tecnológicos fundamentales para planear su integración en el sistema educativo, y los proyectos TIC definen los fundamentos pedagógicos y didácticos para hacer posible esta integración.

Finalmente, se concluye que para que exista una integración exitosa de las TIC en el currículo y se cuente con resultados de aprendizaje alentadores, se hace necesario que las instituciones reevalúen sus prácticas educativas desde su concepción, planeación y ejecución, abran sus puertas a la innovación científica y tecnológica e incorporen los saberes y las experiencias que circulan en la cotidianidad, lo que permitirá impartir un conocimiento contextualizado, garantizar una educación de calidad y formar profesionales competentes en conocimientos, destrezas y disposiciones para la práctica y para la vida, con habilidades sociales, comunicativas y tecnológicas que fortalezcan su crecimiento y sus capacidades de aprendizaje continuo. Estos cambios en el sistema educativo implican empezar a pensar en el qué, cómo y cuándo de una formación que contemple las dimensiones cognitivas, 
éticas, políticas, sociales y culturales del individuo en una sociedad permeada por los desarrollos tecnológicos y las integre en un currículo pertinente, contextualizado y flexible, siendo el tema curricular uno de los indicadores fundamentales para alcanzar las metas internacionales de desarrollo establecidas por la Cumbre Mundial sobre la Sociedad de la Información (CMSI): "el uso de las TIC para perfeccionar el currículo, mejorar la calidad de los logros educativos y promover reformas educativas" (Unesco, 2009, p. 12).

\section{Referencias}

Aguerrondo, I. (2007). La calidad de la educación: ejes para su definición y evaluación. Recuperado de http://oei.es/calidad2/aguerrondo.htm

Beltrán, F. y Gómez, L. (2011). El plan colombiano para integrar un ecosistema digital. Actas de la V Conferencia ACORN-REDECOM. Recuperado de http://slideshare.net/ radarik/el-plan-colombiano-para-integrar-un-ecosistema-digital

Brousseau, G. (2007). Iniciación al estudio de la teoría de las situaciones didácticas. Argentina: Libros del Zorzal.

Ministerio de Educación nacional. (1994). Ley 115, por el cual se reglamenta la ley general de Educación. Recuperado de http://mineducacion.gov.co/1621/articles-85906_archivo_pdf.pdf

Ministerio de Tecnologías de la Información y las Comunicaciones. (1999). Programa Compartel. Recuperado de http://compartel.gov.co

Ministerio de Tecnologías de la Información y las Comunicaciones. (2000). Programa Computadores para educar. Recuperado de http://computadoresparaeducar.gov.co/website/es/

Ministerio de Tecnologías de la Información y las Comunicaciones. (2008). Plan Nacional de Tecnologías de la Información y las Comunicaciones. Recuperado de http://eduteka.org/pdfdir/ColombiaPlanNacionalTIC.pdf

Ministerio de Tecnologías de la Información y las Comunicaciones. (2011). Plan Vive Digital Colombia. Recuperado de http://vivedigital.gov.co/files/Vivo_Vive_Digital.pdf

Ministerio de Educación Nacional. (1998). Indicadores de logros curriculares. Recuperado de http://mineducacion.gov.co/1621/articles-89869_archivo_pdf11.pdf

Ministerio de Educación Nacional. (1998). Lineamientos curriculares del área de Matemáticas. Recuperado de http://mineducacion.gov.co/1621/articles-89869_archivo_pdf9.pdf 
Ministerio de Educación Nacional. (2001). Proyecto incorporación de nuevas tecnologías al currículo de Matemáticas de la Educación Media de Colombia, memorias del seminario nacional. Bogotá: Enlace Editores Ltda.

Ministerio de Educación Nacional. (2002). Decreto 2030. Recuperado de http://mineducacion.gov.co/1621/articles-103106_archivo_pdf.pdf

Ministerio de Educación Nacional. (2005). Programa a que te cojo ratón. Recuperado de http://colombiaaprende.edu.co/html/home/1592/article-99329.html

Ministerio de Educación Nacional. (2006). Estándares básicos de competencias en Matemáticas. Recuperado de http://eduteka.org/pdfdir/MENEstandaresMatematicas2003.pdf

Ministerio de Educación Nacional y Universidad de los Andes. (2007). Programa PlanEsTIC. Recuperado de http://comunidadplanestic.uniandes.edu.co/AcercadePlanEsTIC.aspx

Ministerio de Educación Nacional. (2008). Programa Temáticas. Recuperado de http://colombiaaprende.edu.co/html/docentes/1596/propertyvalue-37838.html

Rico, L. (1998). Complejidad del currículo de matemáticas como herramienta profesional. Revista Latinoamericana de Investigación en Matemática Educativa, 1, 22-39.

Unesco. (1998). Informe Mundial sobre la Educación: los docentes y la enseñanza en el mundo en mutación. Recuperado de http://uned.es/reec/pdfs/04-1998/14_unesco.pdf

Unesco. (2009). Medición de las tecnologías de la información y la comunicación (TIC) en la educación, manual del usuario. Recuperado de http://unesdoc.unesco.org/ images/0018/001883/188309s.pdf

Verillon, P. y Rabardel, P. (1995). Cognitions and artifacts: a contribution to the study ofthought in relation to instrument activity. Revista Europea de Psicología de la Educación, 10(1), 77-101.

Vygotsky, L. (1979). El desarrollo de los procesos psicológicos superiores. Silvia Furió (trad.). Barcelona: Crítica, D. L. 